Pathologe 2014 · 35:143-151

DOI 10.1007/s00292-013-1846-2

Online publiziert: 14. März 2014

c) Springer-Verlag Berlin Heidelberg 2014

Schwerpunktherausgeber

S. Ihrler, München

A. Agaimy, Erlangen
S. Ihrler ${ }^{1,2} \cdot$ O. Guntinas-Lichius ${ }^{3} \cdot$ M. Mollenhauer ${ }^{4}$

${ }^{1}$ Labor für Dermatohistologie und Oralpathologie, München

2 Pathologisches Institut, Ludwig-Maximilians-Universität, München

${ }^{3}$ Hals-, Nasen- und Ohrenklinik, Universität Jena

${ }^{4}$ Institut für Pathologie, Technische Universität, München

\section{Das visionäre Konzept des „Lymphoepithelioms“ 1921 und A. Schmincke}

\section{Nachfolgende terminologische Verwirrung und aktuelle Lösungsansätze}

mie und zur allgemeinen Pathologie, am Ende seiner Tätigkeit in München verfasst, konnte damit kaum auf früheren Arbeiten aufbauen [1].

In dieser deutschsprachigen Arbeit, betitelt „Über lymphoepitheliale Geschwülste“, postulierte Schmincke eine völlig neue Karzinomentität, die er als eindeutig unterschiedlich vom schon gut bekannten klassischen, verhornenden
Plattenepithelkarzinom erachtete [31]. Der Begriff „lymphoepitheliale Geschwülste“ sollte auch zum Ausdruck bringen, dass sich diese Karzinomentität von den verschiedenen lymphoepithelialen Organen

In Auszügen vorgetragen bei der Jahrestagung der Deutschen Gesellschaft für Pathologie in Leipzig, 2011 und beim European Congress of Pathology in Helsinki, 2011. deren deskriptiver Terminologie sich über Jahrzehnte, z. T. bis heute, eine erhebliche Konfusion entwickelt hat (lymphoepithelial, undifferenziert, basaloid, transitional, nichtkeratinisierend, Lymphoepitheliom Typ Schmincke/Typ Regaud und weitere). Ziel der vorliegenden Übersicht ist es, über eine Analyse der historischen Entwicklung zu klären, welche Faktoren zu dieser terminologischen Verwirrung geführt haben.

\section{Alexander Schmincke: Das Konzept des Lymphoepithelioms}

Bis 1920 existierte kein tragfähiges Konzept für die Klassifikation maligner Tumoren im Nasopharynx [35]. Noch bis 1929 wurde für Karzinome des Nasopharynx der damals sehr populäre Begriff „Endotheliom“ verwendet [12]. Die wegweisende Publikation von Alexander Schmincke aus dem Jahr 1921 in der Zeitschrift Beiträge zur pathologischen Anato-

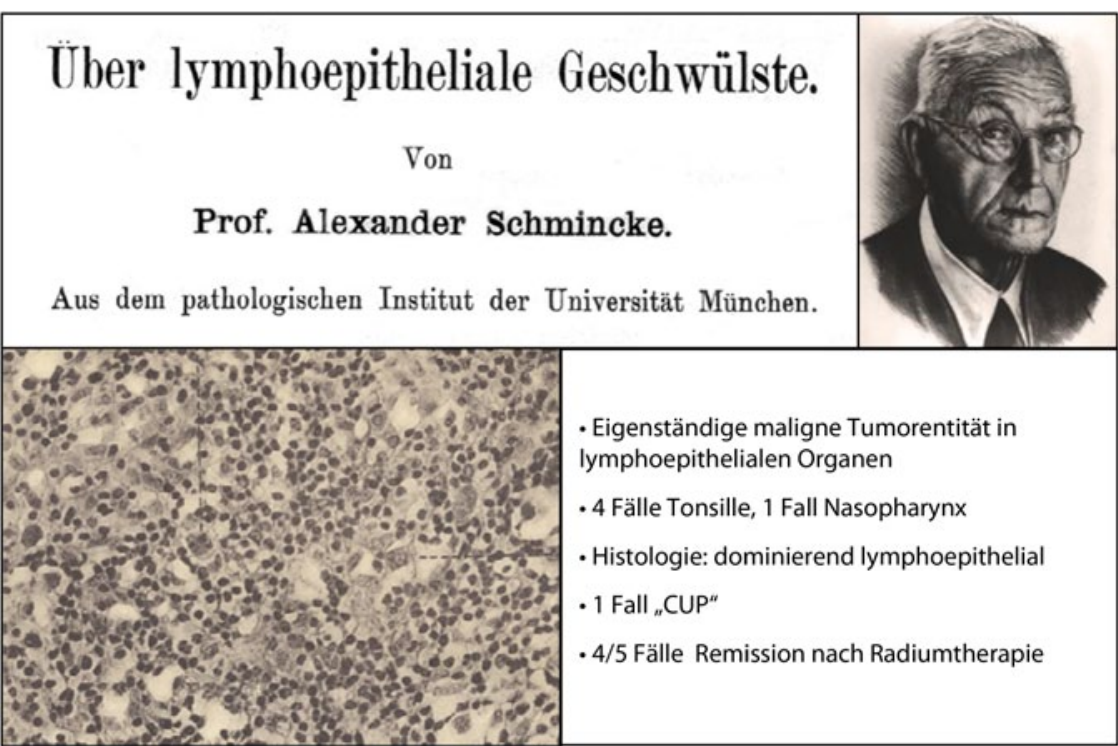

Abb. $1 \Delta$ Deutschsprachige Publikation von Alexander Schmincke in Beiträge zur pathologischen Anatomie und zur allgemeinen Pathologie von 1921 [31]. Das histologische Bild ist gut vereinbar mit einem Tonsillenkarzinom mit starker lymphozytärer Durchsetzung. (Mit freundl. Genehmigung des G.-Fischer-Verlags sowie von Herrn Prof. Dr. P. Schirmacher, Direktor des Instituts für Pathologie der Universität Heidelberg) 


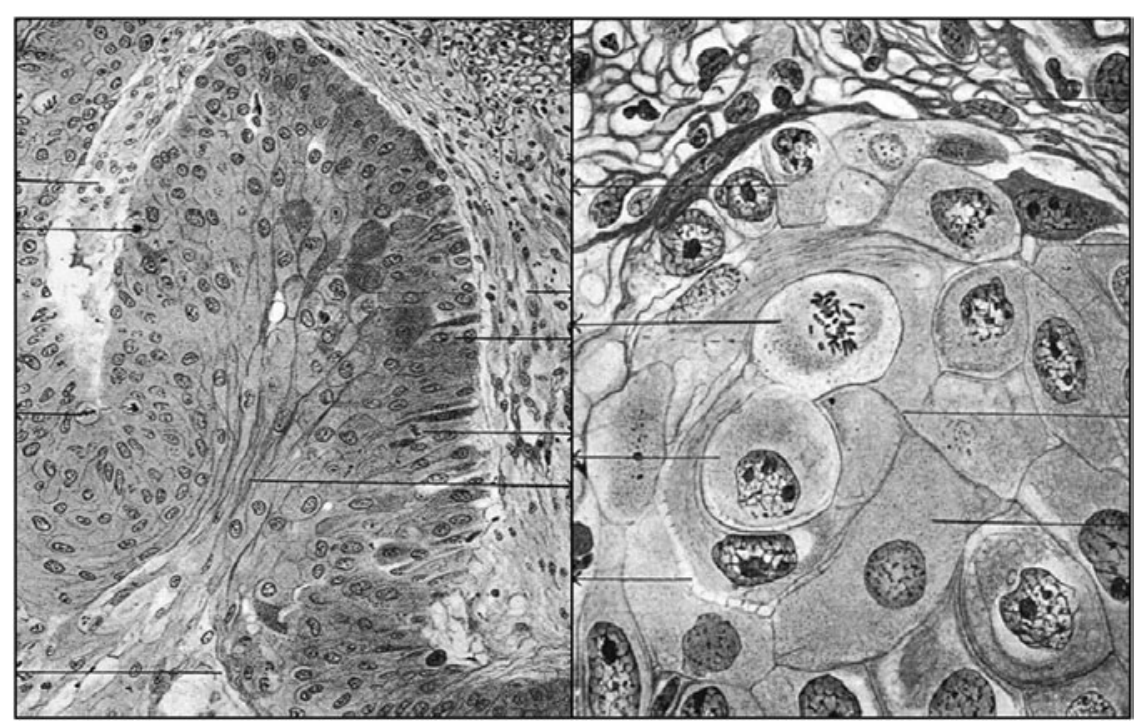

Abb. 2 A Fallbericht der Arbeitsgruppe um Regaud und Reverchon 1921 [27]. Die histologischen Abbildungen zeigen ein Karzinom aus dem Kieferhöhlenbereich mit konventioneller squamöser Differenzierung („Epithelioma epidermoide“). (Mit freundl. Genehmigung von Revue de Laryngologie)

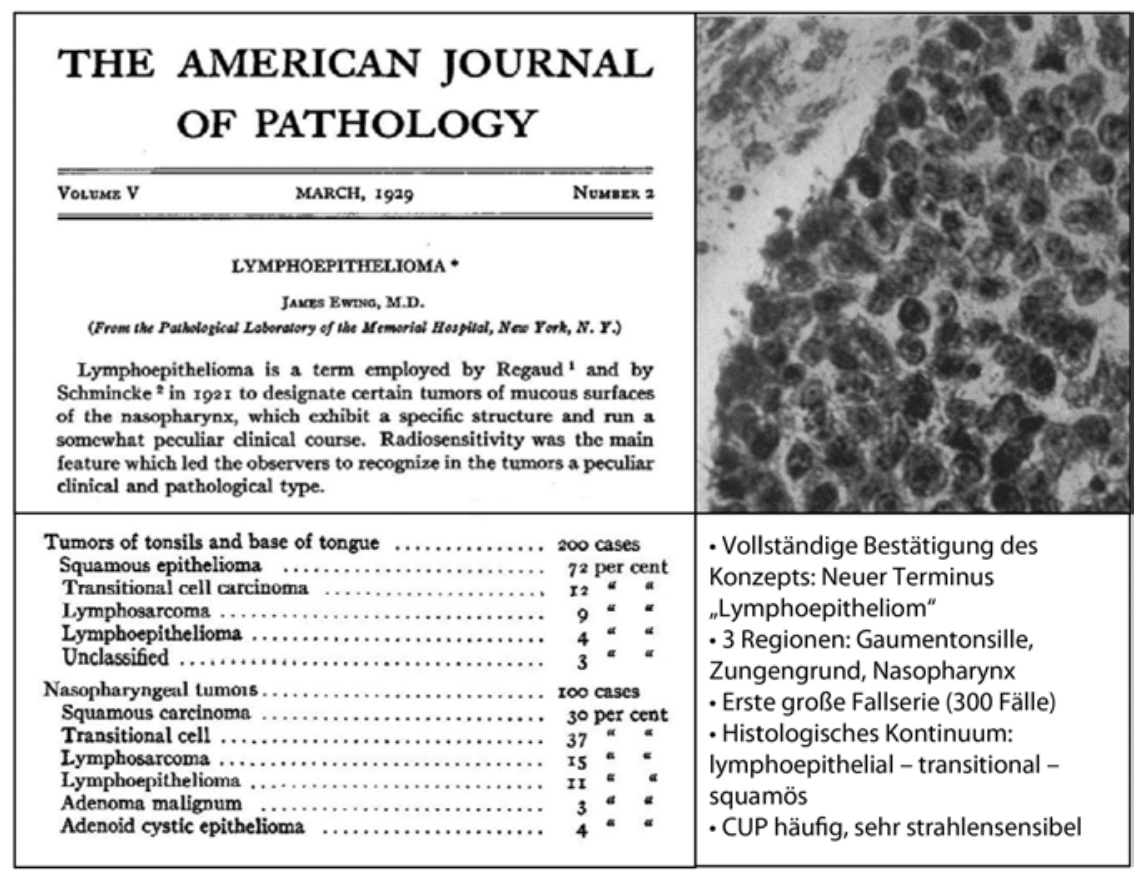

Abb. $3 \Delta$ Publikation von Ewing [11]. Er führt den Begriff "Lymphoepithelioma" ein und bezeichnet im Einleitungssatz Regaud und Schmincke als Erstbeschreiber. Das histologische Bild bezeichnet er gut nachvollziehbar mit "transitional cell carcinoma of the tonsil". (Mit freundl. Genehmigung des Verlags Elsevier)

des Waldeyer-Rachenrings ableitet. Er beschrieb - in detaillierten Fallbeschreibungen und in nach heutigen Kriterien gut nachvollziehbaren Abbildungen - ein Spektrum einer ungewöhnlichen, nichtverhornenden histologischen Differenzierung, ,deren Eigentümlichkeit in einer innigen Verbindung blastomatös wuchernden Epithels mit Lymphocyten rapie, dass trotz einer „starken Proliferationstendenz insofern eine relativ günstige Prognose, als [...] therapeutische Röntgen- und Radiumbestrahlung [...] zur klinischen Geschwulstfreiheit geführt hat". Einer seiner 5 beschriebenen Fälle hatte sich 2 Jahre vor Auftreten des Primärtumors als Lymphknotenmetastase manifestiert, welche erfolgreich bestrahlt wurde - eine Konstellation, die heute als CUP-Syndrom (Cancer of Unknown Primary) bekannt ist. Mit diesem visionären klinisch-histomorphologischen Konzept identifizierte Schmincke die wichtigsten histologischen, klinischen und therapeutischen Besonderheiten, die heute als typisch für virusassoziierte Karzinome im Kopf-Hals-Bereich gelten.

In der z. T. bis heute andauernden Wahrnehmung durch die spätere Literatur wurde der Name Schmincke ausschließlich den Karzinomen des Nasopharynx zugerechnet. Eine Überraschung bei unserer Lektüre der Originalarbeit von A. Schmincke war, dass es sich bei 4 seiner 5 Fälle um Tonsillenkarzinome und nur in einem Fall um ein Nasopharynxkarzinom gehandelt hat. Die Reduzierung von Schminckes Fallserie in der darauf folgenden Literatur auf den Nasopharynx beruhte mutmaßlich darauf, dass meist nicht seine Originalarbeit, sondern Sekundärliteratur herangezogen wurde.

Im gleichen Jahr 1921 publizierte die Pariser Arbeitsgruppe um Claude Regaud in der französischen Zeitschrift Revue de Laryngologie, d' Otologie et de Rhinologie in 2 Fallberichten über die erfolgreiche Strahlentherapie bei KopfHals-Karzinomen [27, 28]. Aus heutiger Sicht zählen beide Fälle allein aufgrund der Lokalisation nicht zum Spektrum lymphoepithelialer Geschwülste, wie von Schmincke publiziert. Beim ersten Fall eines Tumors in der Kieferhöhle, von Regaud bezeichnet als „Epithelioma epidermoide“, zeigt die Betrachtung der histologischen Illustrationen eine konventionelle squamöse Differenzierung (- Abb. 2). Der zweite Fall, lokalisiert im Hypopharynx, wurde von den Autoren zwar als "Lympho-Epitheliome“ bezeichnet, eine histologische Abbildung ist in diesem Fall jedoch leider nicht überliefert. Regaud war als Directeur du Laboratoire de Radiotherapie der Universität Pa- 
Hier steht eine Anzeige.

黛 Springer 
ris nach heutigem Verständnis Onkologe bzw. Strahlentherapeut und fokussierte in seinen beiden Falldarstellungen auf den Erfolg der damals neuen Strahlentherapie und führte keinen neuen Tumortypus an.

\section{Unterstützung für das Konzept des Lymphoepithelioms}

Der berühmte amerikanische Tumorpathologe James Ewing bestätigte und unterstützte 1929 in einem Artikel in The American Journal of Pathology vollständig das Konzept von Schmincke und gab der neuen Tumorentität den Begriff „Lymphoepithelioma" als englische Version von Schminckes Terminus „lymphoepitheliale Geschwülste" [11]. In einer für die damalige Zeit enormen Fallzahl von 300 Tumoren bestätigte Ewing die Gaumentonsille, den Zungengrund und den Nasopharynx als Hauptlokalisationen. Er beschränkte den Terminus des "lymphoepithelioma“ dezidiert nicht auf eine lymphoepitheliale histologische Differenzierung im engeren Sinn, sondern beschrieb ein histologisches Spektrum einer lymphoepithelialen, transitionalen bis zu squamösen Differenzierung. Dazu berichtete er über eine häufige initiale Präsentation mit Lymphknotenmetastasen ohne manifesten Primärtumor (CUP) sowie eine ungewöhnlich gute Radiosensitivität (• Abb. 3).

Ewing benannte im ersten Satz der Einleitung Schmincke und Regaud als Erstbeschreiber dieser Tumorentität; ein Zusammenhang, der vermutlich auf dem von beiden Autoren verwendeten Begriff des „Lymphoepithelioms“ und der jeweils berichteten guten Radiosensitivität beruhte. Er legte mit dieser Namensassoziation in seiner häufig zitierten Publikation die Grundlage für den späteren Terminus „Lymphoepithelioma SchminckeRegaud“ (• Abb.4).

In den folgenden Jahrzehnten unterstützte eine Reihe von Autoren das Konzept des "Lymphoepithelioms“. Neben anderen $[2,3,22]$ sind besonders Publikationen von Doerr [8], Schüler von Schmincke und sein Nachfolger in Heidelberg, sowie Döhnert (• Abb.4; [9]) zu nennen. Noch 1983 erschien im amerikanischen Cancer Journal for Clinicians eine wortgetreue englische Übersetzung der deutschen Original-

Pathologe 2014 - 35:143-151 DOI 10.1007/s00292-013-1846-2

c) Springer-Verlag Berlin Heidelberg 2014

S. Ihrler $\cdot 0$. Guntinas-Lichius · M. Mollenhauer

Das visionäre Konzept des „Lymphoepithelioms" 1921

und A. Schmincke. Nachfolgende terminologische Verwirrung und aktuelle Lösungsansätze

\section{Zusammenfassung}

Mit dem Konzept einer kombiniert klinischhistomorphologisch definierten Karzinomentität, lokalisiert in lymphoepithelialen Organen, begründete Alexander Schmincke vor etwa 90 Jahren eine Tumorentität, welche heute in etwa die Kombination aus nichtkeratinisierendem, häufig humane-Papillomviren(HPV)-assoziiertem Oropharynxkarzinom und nichtkeratinisierendem, meist EpsteinBarr-Virus(EBV)-assoziiertem Nasopharynxkarzinom umfasst. Der Terminus des "Lymphoepithelioms" war ursprünglich von A. Schmincke und J. Ewing als kombinierte klinische und histomorphologische Tumorentität der lymphoepithelialen Organe definiert worden. Die Hauptursache der jahrzehntelangen terminologischen Konfusion ist darin begründet, dass die spätere Beschränkung dieses Terminus auf einen allein histologisch definierten Aspekt (lymphoepitheliale Differenzierung) zu einem nicht praktikablen und nicht sinnvollen Ausschluss von transitional bzw. basaloid differenzierten Karzinomen führte.

Für die kommende WHO-Klassifikation wurde vorgeschlagen, Plattenepithelkarzino- me im Kopf-Hals-Bereich nicht mehr wie bisher allein nach der prognostisch nur sehr eingeschränkt aussagefähigen histologischen Differenzierung, sondern wegen der damit verbundenen klinischen Relevanz für Prognose und Therapie primär nach ätiopathogenetischen Gesichtspunkten zu klassifizieren (klassische Noxen, HPV, EBV). Dies wäre eine eindrucksvolle Bestätigung von Schminckes visionärem Konzept einer klinisch-histomorphologisch definierten Tumorentität, mit dem er seiner Zeit konzeptionell weit voraus war. Gleichzeitig sollte damit das terminologische Dilemma des schlecht reproduzierbaren und prognostisch wenig aussagekräftigen histologischen Differenzierungsspektrums in den Hintergrund treten.

Schlüsselwörter

Oropharynxkarzinom .

Nasopharynxkarzinom · Lymphoepitheliales Karzinom · EBV (Epstein-Barr-Virus) · HPV (humane Papillomviren)

\section{The visionary concept of "Iymphoepithelioma" by A. Schmincke in 1921. Subsequent confusion over terminology and current approach to a solution}

\section{Abstract}

In 1921 Alexander Schmincke established the visionary concept of a clinically and histomorphologically defined carcinoma entity of different lymphoepithelial organs that he named "lymphoepithelioma". This nowadays mainly comprises non-keratinizing oropharyngeal carcinomas frequently associated with human papillomavirus (HPV) and non-keratinizing nasopharyngeal carcinomas mostly associated with Epstein-Barr virus (EBV). The term lymphoepithelioma was originally defined by A. Schmincke and J. Ewing as a combined clinical and histological tumor entity of lymphoepithelial organs. The main reason for the longstanding terminological confusion regarding the term lymphoepithelioma is based on the fact that lateron a pure histological interpretation (lymphoepithelial differentiation) caused an artificial and nonreproducible exclusion of tumors with transitional and basaloid differentiation.
For the forthcoming new WHO classification it has been suggested that squamous cell carcinoma of the head and neck should no longer be classified according to the heterogeneous histological differentiation but according to etiopathogenetic criteria (e.g. HPV-related, EBV-related, nicotine and alcohol-related). This proposed classification corresponds much better to the prognosis and therapy and would represent a late acknowledgement of Schmincke's visionary concept of a clinically and histomorphologically defined tumor entity. In addition, the ongoing terminological confusion over the heterogeneous and prognostically weak spectrum of histological differentiation would subside.

\section{Keywords}

Oropharyngeal carcinoma - Nasopharyngeal carcinoma - Lymphoepithelial carcinoma . Epstein Barr virus · Human papillomavirus 


\section{Lymphoepithelioma Schmincke-Regaud \\ GöTZ DöHNERT \\ Institute of Pathology, University of Heidelberg (Head: Prof. Dr. W. Doerr) \\ Received November 11, 1970}

Abb. $4 \Delta$ Fallbericht von Döhnert: Beispiel für den häufig verwendeten Terminus "Lymphoepithelioma Schmincke-Regaud". (Nach [9])

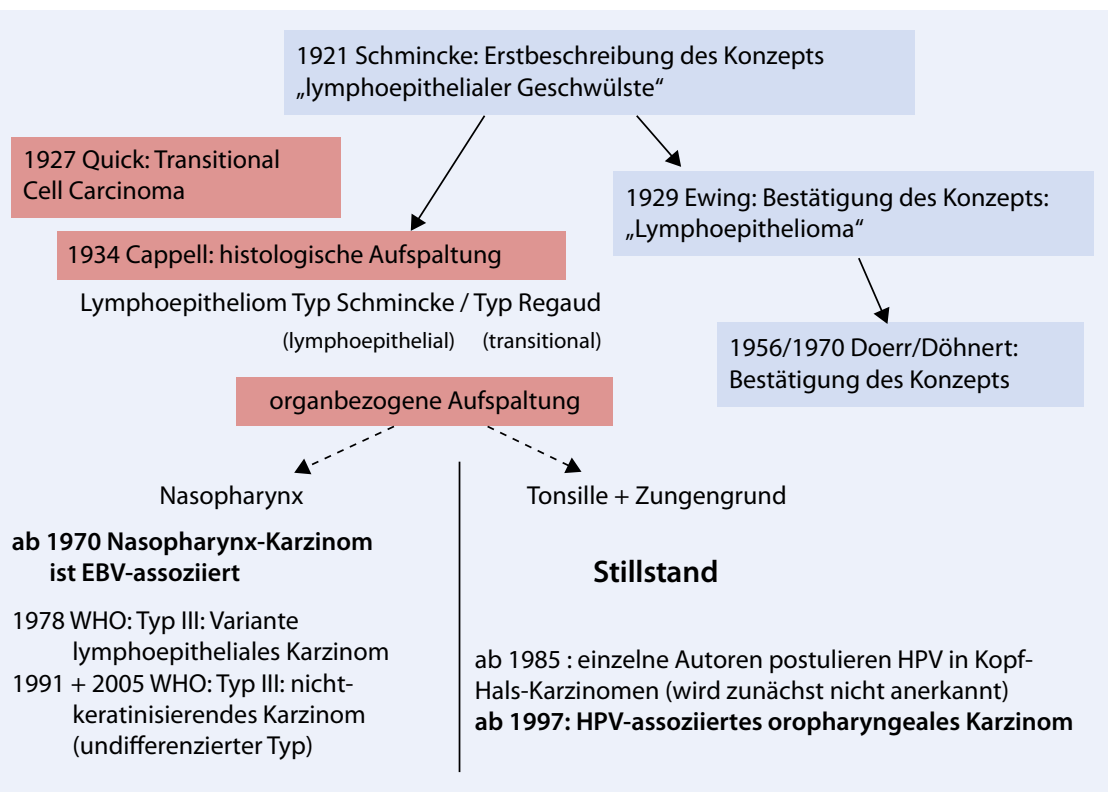

Abb. $5 \Delta$ Zeitliche Abfolge der Erstbeschreibung und Unterstützung (blau) sowie Infragestellung (rot) des Konzepts „Iymphoepitheliale Geschwülste“. EBV Epstein-Barr-Virus, HPV humaner Papillomvirus

\section{Undifferentiated Carcinoma of Nasopharyngeal Type of Tonsil}

Jerzy Klijanienko, MD; Christian Micheau, MD; Nacer Azli, MD; Esteban Cvitkovic, MD; François Esehwege, MD; Patrick Marandas, MD; Jean-Pierre Armand, MD; Odile Casiraghi, MD; Guy Schwaab, MD; Florent de Vathaire, PhD

Abb. $6 \Delta$ Beispiel für die Begriffskonfusion: Der Mangel an geeigneten Begriffen für den Oropharynx verleitet zur kuriosen Verwendung des Terminus des undifferenzierten Nasopharynxkarzinoms für einen Tonsillentumor. (Nach [16])

publikation von A. Schmincke, im Vorwort angekündigt als „landmark work“ [30]. Dies war ein sehr ungewöhnlicher Vorgang. Er illustrierte die fortdauernde Wertschätzung und wissenschaftliche Aktualität von Schminckes Konzept noch 62 Jahre nach seiner deutschsprachigen Publikation. Zusätzlich ist dies auch ein Ausdruck für die hohe Wertschätzung der klassischen deutschen Pathologie im angloamerikanischen Sprachraum.

\section{Begriffliche Konfusion durch Fokussierung auf Teilaspekte}

Parallel zur großen Unterstützung für das Konzept des Lymphoepithelioms kam es zu Entwicklungen, die dieses Konzept nach und nach in Frage stellten bzw. in Vergessenheit geraten ließen. Dies geschah weniger durch direkte Ablehnung des Konzepts, sondern vielmehr durch Fokussierung auf Einzelaspekte und da- mit einhergehendem Verlust des Gesamtkontexts (- Abb.5). So beschrieben Quick u. Cutler [26] im Jahr 1927 in der Arbeit „Transitional cell epidermoid carcinoma: a radiosensitive type of intraoral tumor" ohne Bezug auf Schminckes Arbeit ein Teilkollektiv dieser Tumorentität mit Beschränkung auf transitional differenzierte Karzinome.

In der Publikation „On Lympho-Epithelioma of the Nasopharynx and Tonsils“ unterstützte Cappell [4] 1934 zunächst vollständig Schminckes Konzept, schlug dann aber vor, Fälle mit eher lymphoepithelialer Differenzierung als „Lymphoepitheliom, Typ Schmincke" und Fälle mit eher transitionaler Differenzierung als „Lymphoepitheliom, Typ Regaud“ zu unterscheiden. Ganz am Ende schränkte er zwar ein, dass dies eigentlich nicht funktioniere: „the separation is very indefinite and frequently impossible“. Mit diesem Vorschlag hatte er eine rein histologisch-deskriptiv definierte, nicht praktikable Aufspaltung des ursprünglich klinisch-morphologisch definierten Konzepts von Schmincke in die Welt gesetzt. Diese Unterscheidung wurde über Jahrzehnte fortgeführt bzw. zitiert, obgleich viele Autoren immer wieder betonten, dass sie in der Praxis nicht reproduzierbar sei $[3,8,9,16]$. Gerade diese histologische Aufspaltung in „Lymphoepitheliom, Typ Schmincke“ und „Typ Regaud“ war ein entscheidender Faktor für die jahrzehntelange begriffliche Konfusion bei diesen Tumoren (• Infobox 1).

Für diese Verwirrung spielte auch die Tatsache eine Rolle, dass die deutsch- bzw. französischsprachigen Publikationen von Schmincke und Regaud für englischsprachige Autoren schlecht zugänglich waren. Des Weiteren blieb die Grenzziehung zwischen atypisch differenzierten Karzinomen und manchen malignen Lymphomen und Sarkomen ohne die Hilfe heutiger immunhistologischer Nachweismethoden in vielen Fällen schwierig, was die Entwicklung einer sinnvollen Terminologie weiter behinderte. So finden sich in der historischen Literatur zahlreiche Abbildungen, bei denen eine sichere Linienzuordnung spekulativ bleiben muss $[3,4,9,11]$. 


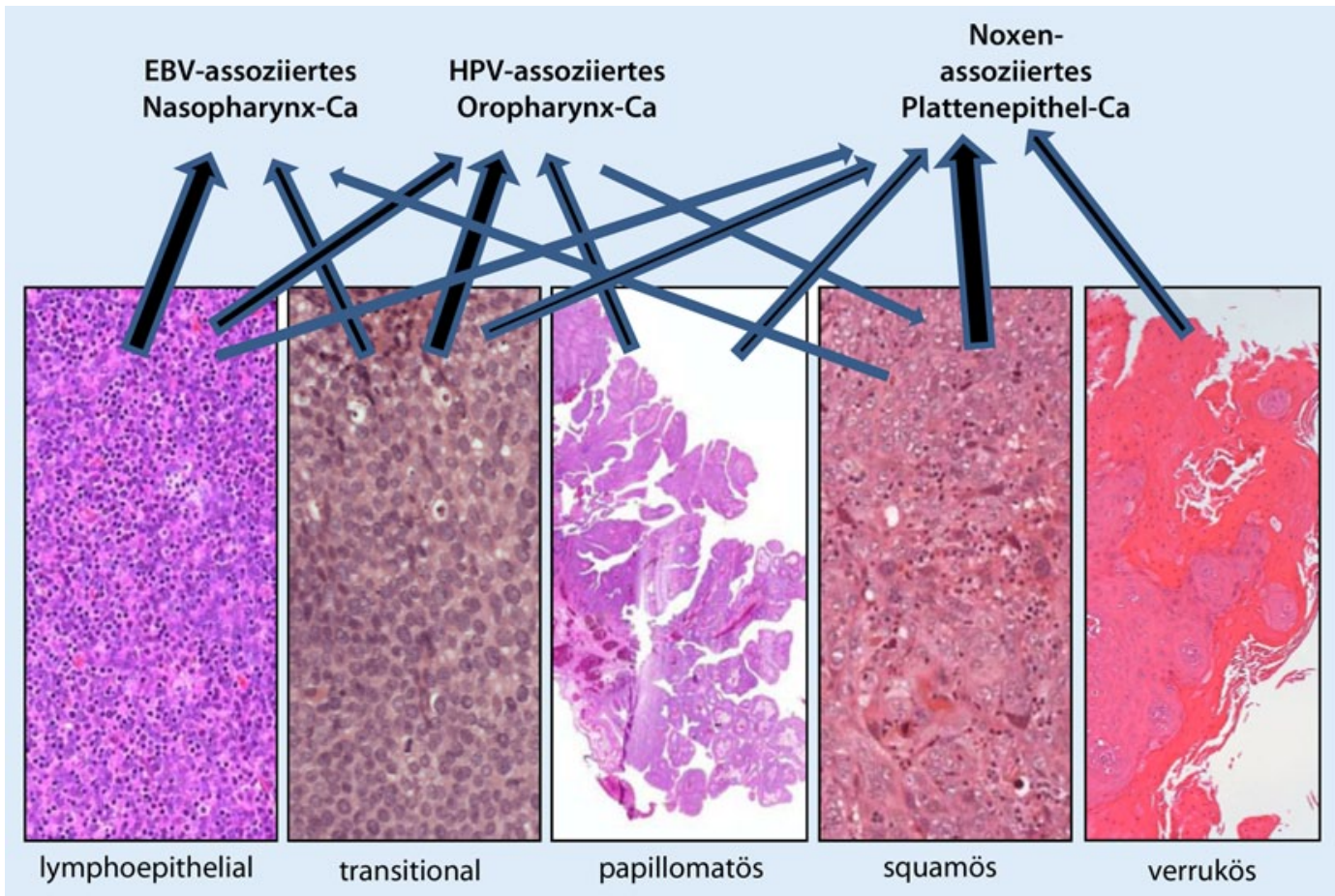

Abb. $7<$ Der postulierte Paradigmenwechsel: zukünftig ätiopathogenetisch definierte Karzinomentitäten anstatt rein histologisch definierter, stark überlappender und deshalb schlecht reproduzierbarer Karzinomentitäten? EBV Epstein-Barr-Virus, HPV humaner Papillomvirus

\section{Fortschritt bei Nasopharynx- karzinomen durch Entdeckung von EBV}

Neben dieser „histologischen Aufspaltung" des Konzepts des Lymphoepithelioms kam es ab etwa 1970 zu einer „Organaufspaltung“(• Abb.5). Auslöser war die Entdeckung, dass das Epstein-Barr-Virus (EBV) eine kausale Bedeutung für das Nasopharynxkarzinom besitzt [14, 41]. Dies führte zu enormen Forschungsaktivitäten und in der Folge zu einem verbesserten Verständnis der Pathogenese sowie einer international anerkannten und gut reproduzierbaren Nomenklatur des Nasopharynxkarzinoms [5, 23, 24, 32, 33]. In der ersten WHO-Klassifikation von 1978 beinhaltete der Typ III (undifferenzierter Typ) des Nasopharynxkarzinoms noch den Subtyp des "Lymphoepithelialen Karzinoms" [33]. Wegen mangelnder Reproduzierbarkeit wurde dieser Subtyp ab der zweiten WHO-Klassifikation 1991 abgeschafft [32].

\section{Stillstand bei Oropharynxkarzinomen}

Nach der Entdeckung der Assoziation des Nasopharynxkarzinoms mit EBV wurde schnell festgestellt, dass EBV bei Karzino- men des Oropharynx und anderer KopfHals-Regionen pathogenetisch keine Rolle spielt. Löning et al. [20] und zur Hausen [40] postulierten 1985 und 1987, nur wenige Jahre nach der Entdeckung des humanen Papillomvirus, dass HPV auch bei einem Teil der Kopf-Hals-Karzinome eine Rolle spielen könnte. Obgleich dies in der Folge immer wieder postuliert wurde, so in Deutschland von Niedobitek et al. [25], konnte sich ein solches Konzept lange nicht durchsetzen (• Abb. 5).

Über Jahrzehnte verursachten der Mangel an wissenschaftlichen Neuentwicklungen und das Fehlen einer adäquaten Nomenklatur eine enorme terminologische Konfusion in Bezug auf die Oropharynxkarzinome $([5,22]$, - Infobox 1). Beispielhaft zeigt $\bullet$ Abb.6, dass in Ermangelung brauchbarer Begriffe sogar die Entität des undifferenzierten Nasopharynxkarzinoms als Ersatzbegriff für histologisch analoge Oropharynxkarzinome herangezogen wurde [16].

Den Höhepunkt der terminologischen Konfusion steuerte die WHO 2005 selbst bei mit der allein deskriptiv-histologischen Neudefinition eines „Lymphoepithelialen Karzinoms" im Kapitel „Tumours of the oral cavity and oropharynx" [36], obgleich die WHO selbst bereits 1991 den gleichlautenden Subtyp des lymphoepithelialen Karzinoms des Nasopharynx wegen mangelnder Reproduzierbarkeit abgeschafft hatte. Ärgerlich an dieser 2005 neu definierten Entität ist v. a. die Tatsache, dass die verantwortlichen Autoren die zu dieser Zeit bereits umfangreich publizierte Assoziation atypisch differenzierter Oropharynxkarzinome mit High-risk-HPV übersehen hatten.

\section{Die neue Entität des HPV-assoziierten oropharyngealen Karzinoms und ihr Verhältnis zum Nasopharynxkarzinom}

Seit Ende der 1990er Jahre häuften sich Publikationen v. a. aus den USA über oropharyngeale Karzinome mit klinischen und histologischen Besonderheiten und Assoziation zu High-risk-HPV (s. vorangegangenen Artikel von Mollenhauer et al. [21] in diesem Heft). In Deutschland kam Klussmann et al. [17] das Verdienst $\mathrm{zu}$, die klinisch wichtigen Besonderheiten dieser Tumoren in der HNO-Heilkunde bekannt gemacht zu haben. Seit ca. 2003 forderten zahlreiche Autoren, darunter Klussmann et al., HPV-assoziierte oropharyngeale Karzinome wegen ihrer klinisch-prognostischen und histomorphologischen Besonderheiten als eigen- 


\section{Infobox 1 Synonyme für das Nasopharynxkarzinom}

Aufgelistet ist die verwirrende Vielfalt der Synonyme bzw. historischen Begriffe [36]. Diese Begriffsvielfalt gilt vergleichbar auch für den Oropharynx:

- Lymphoepitheliom

- Lymphoepitheliomähnliches Karzinom

- Lymphoepitheliales Karzinom

- Lymphoepitheliom Typ Schmincke

- Lymphoepitheliom Typ Regaud

- Transitionalzellkarzinom

- Intermediärzellkarzinom

- Anaplastisches Karzinom

- Undifferenziertes Karzinom mit lymphoidem Stroma

- Karzinom mit vesikulären Kernen

- Plattenepithelkarzinom (WHO 1)

- Nichtkeratinisierendes Karzinom (WHO 2)

Undifferenziertes Karzinom (WHO 3)

Infobox 2 Gemeinsamkeiten von HPV- und EBV-assoziierten Kopf-Hals-Karzinomen (im Vergleich zu noxenassoziierten Karzinomen)

- Jüngere Patienten

- Atypisches histologisches Spektrum

- Sehr selten p53-Genmutation

- Häufig frühe lymphogene Metastasierung

Häufige Manifestation als CUP

Hohe Strahlensensitivität

ständige Karzinomentität anzuerkennen [17, 18]. Dieser evidente Zusammenhang zwischen HPV und Oropharynxkarzinomen wurde von der WHO 2005 unverständlicherweise nicht aufgegriffen [36].

Das HPV-assoziierte Oropharynxkarzinom und das EBV-assoziierte Nasopharynxkarzinom verbinden, wie in $\bullet$ Infobox 2 zusammengefasst, große Ähnlichkeiten in klinischer, histomorphologischer, molekularer, prognostischer und vermutlich in Zukunft therapeutischer Hinsicht und damit gemeinsame Unterschiede zum konventionellen, noxenassoziierten Plattenepithelkarzinom [17-19, $21,23,24,39]$. Daraus drängt sich die in der Literatur erstaunlich wenig diskutierte Frage auf, warum sich diese beiden in vielfältiger Weise so ähnlichen, virusassoziierten Karzinomtypen topographisch in unterschiedlichen Organen des Waldeyer-Rachenrings entwickeln bzw. inwieweit es bzgl. der Topographie doch Überlappungen gibt.

Beim EBV gibt es in der Literatur keine gesicherten Beweise für die Existenz EBV-assoziierter Karzinome in Tonsille und Zungengrund. Es gibt lediglich vereinzelte Berichte über EBV-assoziierte Karzinome (meist vom lymphoepithelialen Typ) ganz überwiegend im Hypopharynx bzw. Larynx [13, 15, 29, 38]. Umgekehrt gibt es schon länger Hinweise auf die Existenz HPV-assoziierter Nasopharynxkarzinome. Nach einer neueren $\mathrm{Pu}$ blikation von Lo et al. [19] weisen ca. 5\% aller Nasopharynxkarzinome (alle dem WHO-Typ I zugehörig) eine Assoziation zu High-risk-HPV auf. Die Gründe für die fast komplett unterschiedliche topographische Lokalisation der beiden virusassoziierten Karzinomtypen innerhalb des Waldeyer-Rachenrings mit nur minimaler Überlappung liegen im Dunkeln und stellen eine interessante Herausforderung für die Zukunft dar.

\section{Bestätigung von Schminckes visionärem Konzept}

Die heutige Kenntnis von 2 topographisch unterschiedlich lokalisierten, hinsichtlich vieler Aspekte sehr ähnlichen, jeweils virusassoziierten Karzinomentitäten in den lymphoepithelialen Organen bestätigt eindrucksvoll das bis in die heutige Zeit gültige Konzept von Schminckes Lymphoepitheliom. Rückblickend hat Alexander Schmincke vor über 90 Jahren fast alle klinischen und histomorphologischen Besonderheiten dieser 2 Karzinomentitäten korrekt erstbeschrieben. Dass er dabei in einer einzigen Publikation - ohne Möglichkeit die jeweilige Virusassoziation erkennen zu können - die Charakteristika nicht nur des nichtkeratinisierenden (meist EBV-assoziierten) Nasopharynxkarzinoms, sondern auch des nichtkeratinisierenden (häufig HPV-assoziierten) Oropharynxkarzinoms erstbeschrieben hat, ist in historischer Hinsicht später in Vergessenheit geraten.

Beide virusssoziierten Karzinome zeigen ein Kontinuum von lymphoepithelialer über transitionaler/basaloider bis hin zu angedeutet squamöser Differenzie- rung. Innerhalb dieses Differenzierungsspektrums überwiegt beim Nasopharynxkarzinom die lymphoepitheliale („undifferenzierte“) Differenzierung und beim Oropharynxkarzinom die transitionale/ basaloide Differenzierung (• Abb. 7). Lewis et al. [18] und andere Autoren forderten in den letzten Jahren einen Paradigmenwechsel, nach dem Plattenepithelkarzinome im Kopf-Hals-Bereich nicht mehr wie bisher üblich nach ihrer deskriptiven histologischen Differenzierung, sondern in Zukunft primär nach ätiopathogenetischen Gesichtspunkten (klassische Noxen, HPV, EBV) klassifiziert werden sollten [17, 39]. Grund ist, dass nur eine Einteilung nach ätiopathogenetischen $\mathrm{Ge}$ sichtspunkten eine entscheidende klinische Relevanz hinsichtlich Prognose und Therapie besitzt (• Abb. 7).

Dieser Vorschlag bestätigt wiederum umfänglich A. Schmincke, der sein Konzept des Lymphoepithelioms nicht durch histomorphologische Kriterien allein (atypische, nichtkeratinisierende Differenzierung), sondern vielmehr durch kombinierte klinisch-histomorphologische Kriterien (Lokalisation, CUP, Radiosensitivität, günstige Prognose) begründet hatte [31]. Mit diesem klinisch-morphologischen Ansatz der Definition einer Tumorentität war Schmincke seiner Zeit konzeptionell weit voraus.

Die Hauptursache der späteren jahrzehntelangen terminologischen Konfusion ist darin begründet, dass der Terminus des "Lymphoepithelioms“ ursprünglich von Schmincke und Ewing als klinisch-morphologischer Tumortypus in lymphoepithelialen Organen definiert worden war, die spätere Beschränkung dieses Terminus auf eine alleinig histologisch definierte Entität mit klassischer lymphoepithelialer Differenzierung jedoch zu einem nicht praktikablen und nicht sinnvollen Ausschluss transitional bzw. basaloid differenzierter Karzinome führte. Dieses semantische Dilemma ist aber nicht Schmincke oder Ewing anzulasten. Es ist zu erwarten, dass eine zukünftige Karzinomklassifikation primär nach ätiopathogenetischen Gesichtspunkten das terminologische Problem der sehr heterogenen, schlecht reproduzierbaren und nur bedingt aussagekräftigen histo- 


\section{Infobox 3 Lebenslauf von \\ Alexander Schmincke}

- Geboren 19.09.1877 in Nürnberg
- Studium der Medizin in Marburg und
Würzburg
- 1907 Habilitation in Würzburg
- $1910-21$ A.o. Professur und Prosektor,
LMU München (bei Max Borst)
- $1921-22$ Lehrstuhl Graz
- $1922-28$ Lehrstuhl Tübingen
- $1928-49$ Lehrstuhl Heidelberg
(Schüler:Wilhelm Doerr)
- $1945-47$ Vorübergehendes Arbeitsverbot
wegen Nähe zum NS-Regime
- Gestorben 26.08.1953 in Heidelberg

logischen Differenzierung in den Hintergrund treten lassen wird.

Alexander Schmincke hat laut einer Übersicht von Wilhelm Doerr 112 Originalpublikationen schwerpunktmäßig zu den Themen Tumorpathologie, Tuberkulose und Neuropathologie verfasst [7]. In der Monographie von Georg Dhom, "Geschichte der Histopathologie“, wird Alexander Schmincke mit dem Zitat gewürdigt: „Erstbeschreibung des nach Schmincke benannten Tumors, das lymphoepitheliale Karzinom" [6]. Mit Ausnahme von Würdigungen zum 75. Geburtstag und Nachrufen kurz nach seinem Tod $[7,34,37]$ ist uns eine Aufarbeitung von Schminckes Bedeutung für Tumoren lymphoepithelialer Organe aus heutiger Sicht nicht bekannt. Sein Lebenswerk ist überschattet durch Nähe zum NS-Regime. Er war seit 1933 Mitglied der SA und später der NSDAP. Nach dem Krieg musste er die Leitungsfunktion der Heidelberger Pathologie für 2 Jahre ruhen lassen. Er wurde, v. a. aufgrund der späteren Fürsprache des jüdischen Oberarztes Walter Pagel, für den sich Schmincke eingesetzt hatte, als ",Mitläufer" eingestuft [10]. Infobox 3 fasst wichtige Stationen aus dem Leben von Alexander Schmincke zusammen $[7,34,37]$.

\section{Fazit für die Praxis}

Vermutlich wird die kommende WHOKlassifikation empfehlen, Plattenepithelkarzinome im Kopf-Hals-Bereich nicht mehr wie bisher nach der prognostisch nur eingeschränkt aussagefähigen his- tologischen Differenzierung, sondern in Zukunft primär nach ätiopathogenetischen Gesichtspunkten (klassische Noxen, HPV, EBV) zu klassifizieren. Dies ist begründet durch die damit verbundene klinische Relevanz für Prognose und Therapie.

\section{Korrespondenzadresse}

Prof. Dr. S. Ihrler

Labor für Dermatohistologie und

Oralpathologie

Bayerstr. 69, 80335 München

Ihrler@dermpath-muenchen.de

Danksagungen. Die Autoren danken Frau Dr. Veronique Weiler für die Hilfe bei französischsprachiger Literatur sowie Herrn Dr. Gregor Babaryka für seine medizingeschichtliche Expertise.

\section{Einhaltung ethischer Richtlinien}

Interessenkonflikt. S. Ihrler, U. Guntinas-Lichius, M. Mollenhauer geben an, dass kein Interessenkonflikt besteht.

Dieser Beitrag beinhaltet keine Studien an Menschen oder Tieren.

\section{Literatur}

1. Babaryka G (2008) Das Pathologische Institut der Universität München in der Ära Max Borst von 1910-1946. In: Kraus E (Hrsg) Die Universität München im Dritten Reich. Aufsätze Teil II. Utz, München, S 63-132

2. Bansberg SF, Olsen KD, Gaffey TA (1989) Lymphoepithelioma of the oropharynx. Otolaryngol Head Neck Surg 100:303-307

3. Black JIM (1938) The lympho-epitheliomata. J Laryngol Otol 53:225-245

4. Cappell DF (1934) On lympho-epithelioma of the nasopharynx and tonsils. J Pathol Bacteriol 39:49 64

5. Chan JKC, Bray F, Mccarron P et al (2005) Nasopharyngeal carcinoma. In: Barnes L, Eveson JW, Reichart P, Sidransky D (Hrsg) World Health Organization classification of tumours. Pathology and genetics of head and neck tumors. IARC Press, Lyon, S $85-$ 97

6. Dhom G (2001) Geschichte der Histopathologie: Schmincke A. Springer, Berlin Heidelberg New York, S 759

7. Doerr W (1954) Alexander Schmincke zum Gedächtnis. Zentralbl Allg Pathol 91:1-9

8. Doerr W (1956) Lymphoepithelial Schmincke-Regaud tumors. Arztl Wochensch 11:169-182

9. Dohnert G (1971) Lymphoepithelioma Schmincke-Regaud. Virchows Arch A Pathol Pathol Anat 352:279-284

10. Eckart WU (2006) Die Ära Schmincke. In: Eckart WU, Sellin V, Wolgast E (Hrsg) Die Universität Heidelberg im Nationalsozialismus. Springer, Berlin Heidelberg New York, S 977-980
11. Ewing J (1929) Lymphoepithelioma. Am J Pathol 5:99-108 107

12. Gardham AJ (1929) Endothelioma of the nasopharynx: an infiltration tumour at the base of the skull. Br J Surg 99:242-263

13. Goldenberg D, Benoit NE, Begum S et al (2004) Epstein-Barr virus in head and neck cancer assessed by quantitative polymerase chain reaction. Laryngoscope 114:1027-1031

14. Henle W, Henle G, Ho HC et al (1970) Antibodies to Epstein-Barr virus in nasopharyngeal carcino$\mathrm{ma}$, other head and neck neoplasms, and control groups. J Natl Cancer Inst 44:225-231

15. Khabie N, Savva A, Kasperbauer JL et al (2001) Epstein-Barr virus DNA is not increased in tonsillar carcinoma. Laryngoscope 111:811-814

16. Klijanienko J, Micheau C, Azli N et al (1989) Undifferentiated carcinoma of nasopharyngeal type of tonsil. Arch Otolaryngol Head Neck Surg 115:731734

17. Klussmann JP, Preuss SF, Speel EJ (2009) Human papillomavirus and cancer of the oropharynx. Molecular interaction and clinical implications. HNO 57:113-122

18. Lewis JS Jr, Thorstad WL, Chernock RD et al (2010) p16 positive oropharyngeal squamous cell carcinoma: an entity with a favorable prognosis regardless of tumor HPV status. Am J Surg Pathol 34:1088-1096

19. Lo EJ, Bell D, Woo JS et al (2010) Human papillomavirus and WHO type I nasopharyngeal carcinoma. Laryngoscope 120:1990-1997

20. Loning T, Ikenberg H, Becker J et al (1985) Analysis of oral papillomas, leukoplakias, and invasive carcinomas for human papillomavirus type related DNA. J Invest Dermatol 84:417-420

21. Mollenhauer M, Assmann G, Zengel P et al (2014) HPV-assoziierte oropharyngeale Karzinome: aktueller Stand und das Verhältnis zu CUP. Pathologe, DOI:10.1007/s00292-013-1852-4

22. Möller P, Wirbel R, Hofmann W et al (1984) Lymphoepithelial carcinoma (Schmincke type) as a derivate of the tonsillar crypt epithelium. Virchows Arch A Pathol Anat Histopathol 405:85-93

23. Niedobitek G (1998) Epstein-Barr virus infection in the pathogenesis of nasopharyngeal carcinoma. Pathologe 19:337-344

24. Niedobitek G, Hansmann ML, Herbst H et al (1991) Epstein-Barr virus and carcinomas: undifferentiated carcinomas but not squamous cell carcinomas of the nasopharynx are regularly associated with the virus. J Pathol 165:17-24

25. Niedobitek G, Pitteroff S, Herbst H et al (1990) Detection of human papillomavirus type 16 DNA in carcinomas of the palatine tonsil. J Clin Pathol 43:918-921

26. Quick D, Cutler M (1927) Transitional cell epidermoid carcinoma: a radiosensitive type of intra-oral tumor. Surg Gynecol Obstet 45:320-331

27. Regaud C, Reverchon L (1921) Sur un cas d'épithélioma épidermoide développé dans le massif maxillaire supérieur, étendu aux téguments de la face, aux cavités buccale, nasale et orbitaire, ainsi qu'aux ganglions du cou, guéri par la curiethérapie. Rev Laryngo 41:368-378

28. Reverchon, Coutard (1921) Lympho-épithéliome de l'hypopharynx traité par röntgenthérapie sans réaction notable du pharynx et du larynx. Bull Soc Franc Oto Rhino-lar 34:209-214

29. Rytkonen AE, Hirvikoski PP, Salo TA (2011) Lymphoepithelial carcinoma: two case reports and a systematic review of oral and sinonasal cases. Head Neck Pathol 5:327-334 
30. Schmincke A (1983) On lymphoepithelial tumors. CA Cancer J Clin 33:172-179

31. Schmincke A (1921) Über Lymphoepitheliale Geschwülste. Beiträge zur pathologischen Anatomie und zur allgemeinen Pathologie 68:161-170

32. Shanmugaratnam K, Sobin LH (1991) Histological typing of tumours of the upper respiratory tract and ear. In: Shanmugaratnam K (Hrsg) International histological classification of tumours, 2. Aufl. World Health Organization, Geneva, S 32-33

33. Shanmugaratnam K, Sobin LH (1978) Histological typing of upper respiratory tract tumours. In: Shanmugaratnam K (Hrsg) International histological classification of tumours. World Health Organization, Geneva, S $32-33$

34. Siegmund H (1952) 75th Birthday of Prof. Alexander Schmincke. 18 Sept. 1952. Munch Med Wochenschr 94:1871-1872

35. Trotter W (1911) On certain clinically obscure malignant tumours of the naso-pharyngeal wall. $\mathrm{Br}$ Med J 2:1057-1059

36. Tsang WYW (2005) Lymphoepithelial carcinoma. In: Barnes L, Eveson JW, Reichart P, Sidransky $D(H r s g)$ World Health Organization classification of tumorus. Pathology and genetics of head and neck tumors. IARC Press, Lyon, S 176

37. Wurm H (1955) Alexander Schmincke. Verh Dtsch Ges Pathol 38:393-395

38. Zbaren P, Borisch B, Lang H et al (1997) Undifferentiated carcinoma of nasopharyngeal type of the laryngopharyngeal region. Otolaryngol Head Neck Surg 117:688-693

39. Zengel P, Assmann G, Mollenhauer M et al (2012) Cancer of unknown primary originating from oropharyngeal carcinomas are strongly correlated to HPV positivity. Virchows Arch 461:283-290

40. Zur Hausen H (1987) Papillomaviruses in human cancer. Cancer 59:1692-1696

41. Zur Hausen H, Schulte-Holthausen $\mathrm{H}$, Klein $\mathrm{G}$ et al (1970) EBV DNA in biopsies of Burkitt tumours and anaplastic carcinomas of the nasopharynx. Nature 228:1056-1058

\section{Ergänzung zum Beitrag „Prädik- tive Biomarker tumorrelevanter Signalwege in der molekularen Pathologie"}

Bonzheim I., Fend F. (2014)
Pathologe 35: 93-105

Aufgrund der Zeitverzögerung zwischen Annahme und Veröffentlichung des Beitrags muss zum kolorektalen Karzinom aufgrund neuer Erkenntnisse und geänderter Zulassungsbedingungen für anti-EGFR zielgerichtete therapeutische Antikörper folgende Ergänzung erfolgen:

Eine neue Studie zum kolorektalen Karzinom hat gezeigt, dass neben den KRAS-Kodons 12 und 13 (Exon 2) die Bestimmung der KRASKodons 59, 61 (Exon 3), 117 und 146 (Exon 4) und NRAS-Kodons 12, 13 (Exon 2), 59, 61 (Exon 3), 117 und 146 (Exon 4) eine Grundvoraussetzung für die Verabreichung eines antiEGFR Antikörpers darstellen muss [1]. Ein Teil der kolorektalen Karzinome, die die WildtypSequenz in den KRAS-Kodons 12 und 13 aufweisen, zeigen Mutationen in diesen Kodons des KRAS- und des NRAS-Gens (KRAS-Kodon 61: 3,7\%, Kodon 146: 3,3\%, NRAS-Kodon 12: 1,8\%, Kodon 13: 0,2\%, Kodon 61: 3,1\%; zu KRAS-Kodons 59 und 117, NRAS 59, 117, und 146 keine Angaben [2]). Diese Mutationen führen bei der Anti-EGFR-Antikörper-Therapie zu schlechterem progressionsfreien und Gesamt-Überleben der Patienten als bei Therapieansätzen ohne Verabreichung des Antikörpers. Eine Anti-EGFR-Therapie stellt somit für Patienten mit positivem Mutationsstatus in den Kodons 12, 13, 59, 61, 117 und 146 des KRAS- und des NRAS-Gens einen Nachteil dar und sollte nur im Falle eines negativen Mutationsstatus dieser Kodons verabreicht werden. Diese Angaben beruhen allerdings auf sehr wenigen Studien und können sich zukünftig noch ändern.

Korrespondenzadresse:

Dr. rer. nat. I. Bonzheim

Institut für Pathologie und Neuropathologie, Universitätsklinikum Tübingen und

Comprehensive Cancer Center,

Eberhard-Karls-Universität,

Liebermeisterstraße 8,

72076 Tübingen

irina.bonzheim@med.uni-tuebingen.de

\section{Literatur}

1. Douillard JY, Oliner KS, Siena S, Tabernero J, Burkes R, Barugel M, et al. PanitumumabFOLFOX4 treatment and RAS mutations in colorectal cancer. The New England journal of medicine. 2013 Sep 12;369(11):1023-34

2. Vaughn $C P$, Zobell $S D$, Furtado $L V$, Baker $C L$, Samowitz WS. Frequency of KRAS, BRAF, and NRAS mutations in colorectal cancer. Genes, chromosomes \& cancer. 2011 May;50(5):307-12 